\title{
When Benign Tumors Mimic Malignancies: A Case of Lymphangiomatosis Masquerading as Metastatic Disease
}

Kristen R. Spencer $\cdot$ Markku M. Miettinen •

Robert G. Maki $\cdot$ Janice M. Mehnert

To view enhanced content go to www.rarecancers-open.com

Received: May 10, 2013 / Published online: September 28, 2013

(c) The Author(s) 2013. This article is published with open access at Springerlink.com

\section{ABSTRACT}

Lymphangiomatosis, a rare disorder of the lymphatic system characterized by the abnormal proliferation of lymphatic vessels, is a typically benign disorder that at times can exhibit invasive or malignant behavior. While generally considered a diagnosis of childhood, in adults the majority of cases are asymptomatic and found incidentally. Rarely, lymphatic overgrowth can occur, causing growth of lesions on imaging mimicking a metastatic process and occasionally, resulting in substantial morbidity and mortality. Here, we

\section{K. R. Spencer}

Department of Medicine, Rutgers University-Robert Wood Johnson Medical School, 675 Hoes Lane, Piscataway, NJ 08854, USA

\section{M. Miettinen}

National Cancer Institute Center for Cancer Research, Building 10, Magnuson CC, Room 2B50, 10 Center Drive, Bethesda, MD 20892, USA

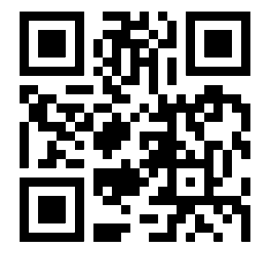

Enhanced content for this article is available on the journal web site:

www.rarecancers-open.com present such a case of lymphangiomatosis with multi-organ system involvement in liver, bone, and spleen. In addition to details of the clinical presentation and the pathologic review which led to the diagnosis, we describe our use of the tyrosine kinase inhibitor pazopanib, which may cause stabilization of lymphangiomatosis through blockade of vascular endothelial growth factor (VEGF) signaling, for systemic treatment in this unusual case.

Keywords: Lymphangiomatosis; Pazopanib; VEGF

\section{R. G. Maki}

Division of Hematology and Medical Oncology, Department of Medicine, Mount Sinai Hospital, Annenberg Building Floor 4, Room 4-52, 1468 Madison Avenue, New York, NY 10029, USA

\section{J. M. Mehnert}

Rutgers Cancer Institute of New Jersey, 195 Little Albany Street, New Brunswick, NJ 08903, USA

\section{J. M. Mehnert ( $\square)$}

Division of Medical Oncology, Department of Medicine, Rutgers University-Robert Wood Johnson Medical School, 675 Hoes Lane, Piscataway, NJ 08854, USA

e-mail: mehnerja@cinj.rutgers.edu 


\section{INTRODUCTION}

Many oncologists will encounter patients whose tumors display benign histology and invasive or malignant behavior. This is particularly relevant as new pathologic entities emerge due to novel discoveries in the field, advances in molecular diagnostics, and tumor reclassifications. One example of a benign entity that can occasionally exhibit aggressive behavior is lymphangiomatosis, a rare disorder of the lymphatic system characterized by the abnormal proliferation of lymphatic vessels, sometimes referred to as generalized lymphatic anomaly. When focal these malformations are termed lymphangiomas, but when widespread the process is referred to as lymphangiomatosis [1-5]. This disorder is histologically benign, as organs are replaced by structurally normal lymphatic channels and cellular components are mature and well differentiated [3]. Lymphangiomatosis is generally a disease of childhood, with most cases being diagnosed by the second decade of life [2-5].

In adults, the majority of cases of lymphangiomatosis are asymptomatic and often found incidentally on imaging ordered for unrelated conditions. Symptomatic disease, while rare, does occur and tends to be caused by compressive effects or organomegaly and the resulting sequelae $[4,5]$. However, lymphatic overgrowth can cause substantial morbidity, and occasionally mortality, through local tissue destruction. Historically lymphangiomatosis has been shown to cause pulmonary, splenic or skeletal disease, though it has also been found in locations such as the intestine, extremities, head and neck, and heart [2-5]. The variable presentation of lymphangiomatosis and the rare incidence of symptomatic cases requiring treatment have made establishing treatment guidelines difficult. The mainstay of treatment continues to be resection when possible for localized disease, with palliation being the goal in cases of diffuse spread [1, 3, 4]. Sclerotherapy and radiotherapy have been tried to no avail, and systemic corticosteroids and chemotherapeutic agents such as cyclophosphamide and cytokine interferon (IFN) have shown either mixed results or had significant side effects [5-15]. There has been some promising anecdotal evidence for the use of angiogenesis and lymphangiogenesis inhibitors such as vascular endothelial growth factor (VEGF) and mammalian target of rapamycin (mTOR) inhibitors. Indeed, clinical benefit was reported in one case of diffuse pulmonary lymphangiomatosis treated with the VEGF inhibitor bevacizumab [1], as well as in an infant treated with the mTOR inhibitor sirolimus [5]. These approaches make scientific sense given the off-target antiangiogenic effects of mTOR inhibitors by way of inhibition of kinase activity resulting in cell death, and the role of VEGF in lymphangiogenesis, which is primarily driven by the interaction of VEGF-C and D isoform signaling through VEGF-receptors 2 and 3, and also by the VEGF-A isoform through VEGF receptor 2 [1]. Here, we present a case of lymphangiomatosis with multi-organ system involvement in an adult with growing lesions that was treated with pazopanib, an orally available potent and selective small molecule tyrosine kinase inhibitor targeting VEGFR 1-3, platelet-derived growth factor receptors (PDGFR) $\alpha / \beta$, and c-kit [16].

\section{CASE REPORT}

The patient was agreeable to having her images and clinical history published in this report. The patient is a 33-year-old female with a past medical history significant only for a complicated twin pregnancy following in vitro fertilization four years prior, who presented to 
the emergency department complaining of chest pain and dyspnea. A computed tomography angiogram (CTA) performed at that time was negative for pulmonary embolism, but found incidental pulmonary nodules without further parenchymal or vascular lung abnormalities, as well as sclerosis of the T7 vertebral body and multiple enhancing lesions in the liver and spleen peripherally. Her dyspnea improved and she followed up with her primary physician as an outpatient. Although her symptoms were likely unrelated to the CTA findings, a triphasic CT of the abdomen and pelvis was repeated and confirmed a sclerotic T7 vertebral body, scattered sclerotic foci throughout the spine, a $9 \mathrm{~mm}$ nodule in the left lower lobe of the lung, multiple hypodense lesions in the liver with the largest $5.5 \times 3.3 \mathrm{~cm}$ in the left lobe, and an enlarged spleen with hypodense lesions measuring $11.4 \times 6.4 \mathrm{~cm}$ in size (see Figs. 1, 2) There was no identifiable adenopathy. A magnetic resonance imaging (MRI) of the abdomen was performed and again showed multiple large hepatic lesions, the largest in the left lobe now $9 \mathrm{~cm}$, and multiple splenic lesions, the largest now $13 \mathrm{~cm}$, that did not demonstrate typical MR characteristics of hemangiomas. Hemogram and chemistry profile were normal. CEA, CA 125, CA 19-9 and alpha fetoprotein levels were all within normal limits.

Upon presentation to us the patient denied any constitutional complaints and her dyspnea had completely resolved. A comprehensive physical exam was pertinent for a palpable spleen but no appreciable hepatomegaly or lymphadenopathy, no tenderness to palpation over her vertebral spine and no other abnormal signs. Because of the uncharacteristic radiographic findings, the sclerotic bony disease and the marked progression in size of

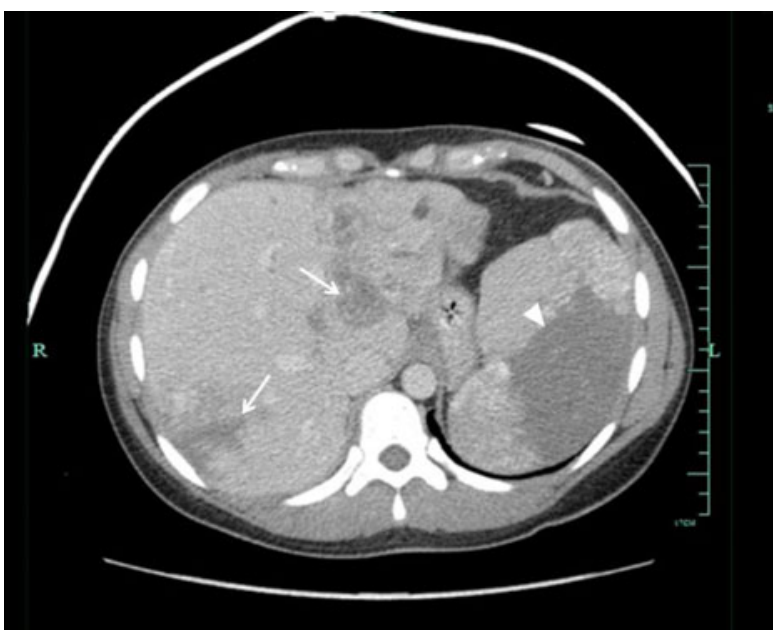

Fig. 1 Initial computed tomography demonstrating the presence of hepatic (arrows) and splenic (arrowhead) lesions consistent with a multifocal or metastatic process

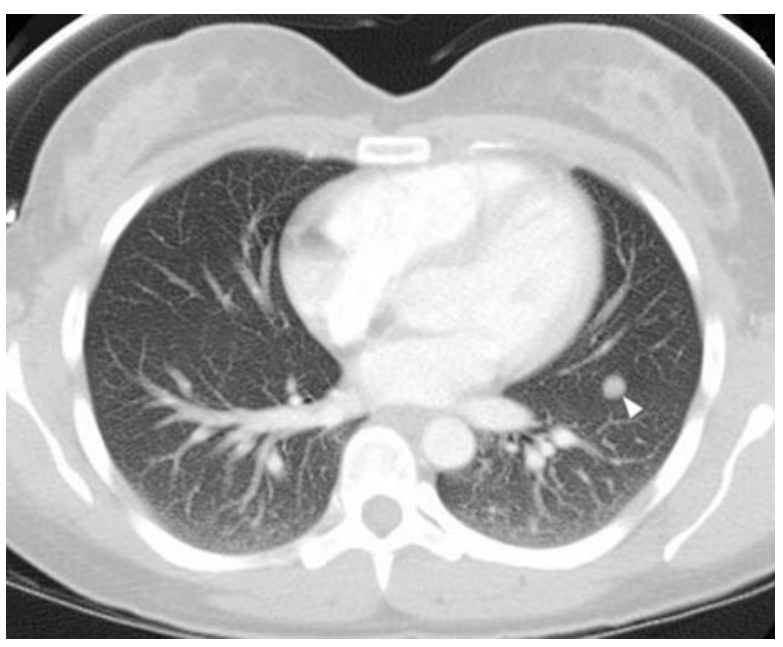

Fig. 2 Initial computed tomography demonstrating the presence of a pulmonary nodule (arrowhead) consistent with a multifocal or metastatic process

the lesions in the short time between scans, the appearance was felt to be overall indicative of a metastatic process and an ultrasound guided liver biopsy was performed. The biopsy showed well-formed vessels embedded in fibrous stroma infiltrating into the hepatic parenchyma. The vessels were lined with flattened, focally enlarged and mildly atypical endothelial cells with focal cytoplasmic vacuolization. They showed immunoreactivity for VIII-related 
antigen, CD31, CD34, and podoplanin (D240), consistent with lymphatic endothelial phenotype. The tumor cells were negative for keratins CK7 and CK8 (CAM5.2), typically positive in epithelial cells. These results supported a diagnosis of a vascular neoplasm with clear cell features and a littoral cell phenotype. The original differential diagnosis included bacillary angiomatosis, though there was no evidence of immunocompromise, and littoral cell angioma because of the histologic phenotype and known case reports of this process to involve both the liver and spleen [17]. Given the clinical features of additional metastatic appearing lesions, however, the diagnosis of a hemangioendothelioma or a low-grade, unusual clear cell angiosarcoma could not be excluded $[17,18]$. At that time, it was felt a second biopsy should be obtained to further characterize the lesions outside the liver, and since several weeks had passed, repeat imaging was ordered to re-assess their behavior.

The patient was again seen in follow-up and the repeat CT of the chest, abdomen and pelvis was obtained which showed further increase in the size of the hepatic lesions, growth of the spleen from 11.5 to $13.6 \mathrm{~cm}$ in greatest dimension and increased central hypodensity in the spleen compatible with necrosis. Both bone and lung lesions were stable. Interim repeat hemogram also showed new thrombocytopenia of 133,000. CT-guided core needle biopsy of the spleen was chosen based on imaging and showed a tumor morphologically similar to the hepatic lesion, with a benign process with clear cell changes involving both specimens. Both hepatic and splenic specimens were sent for additional peer review at that time. It was noted there that the vascular profiles often contained proteinaceous fluid and lymphocytes supporting lymphatic vascular-type proliferation, with focal endothelial hobnailing. The lymphatic endothelial cells were again positive for CD31, CD34, and podoplanin but negative for keratin 8 (CAM 5.2), keratin 7, and CD45 (Fig. 3). Given that the morphology and immunophenotype supported the diagnosis of lymphangiomatosis, and that this process may involve liver, spleen and bone as well as exhibit growth in rare cases, it was agreed that this diagnosis was most accurate.

With the observed short-term growth and necrosis particularly in the spleen, as well as developing thrombocytopenia, systemic therapy was recommended for this patient. Pazopanib, a potent and selective tyrosine kinase inhibitor of VEGF-receptors 1, 2, and 3 as well as KIT and PDGF receptors, was chosen based on the initial possible working diagnoses of low-grade angiosarcoma versus a metastatic littoral cell tumor of the spleen, its kinase profile (active against VEGF signaling) and regulatory approval in advanced sarcomas. The patient was started on $800 \mathrm{mg}$ of pazopanib by mouth daily. Although no reduction in the size of the lesions was noted, growth of the splenic and liver lesions stabilized on follow-up imaging and the patient's hemogram normalized. The disease remains stable after one year of therapy. The patient has experienced minimal treatment related side effects consisting of minimal nausea, and is clinically well at this time.

\section{DISCUSSION}

As clinicians, questions of monitoring and treatment are raised by cases of "malignant masqueraders" such as the one above. Indeed, the presentation of lymphangiomatosis with multi-organ involvement has been mistaken for advanced, metastatic cancers such as melanoma [4]. The lesions observed in this patient 

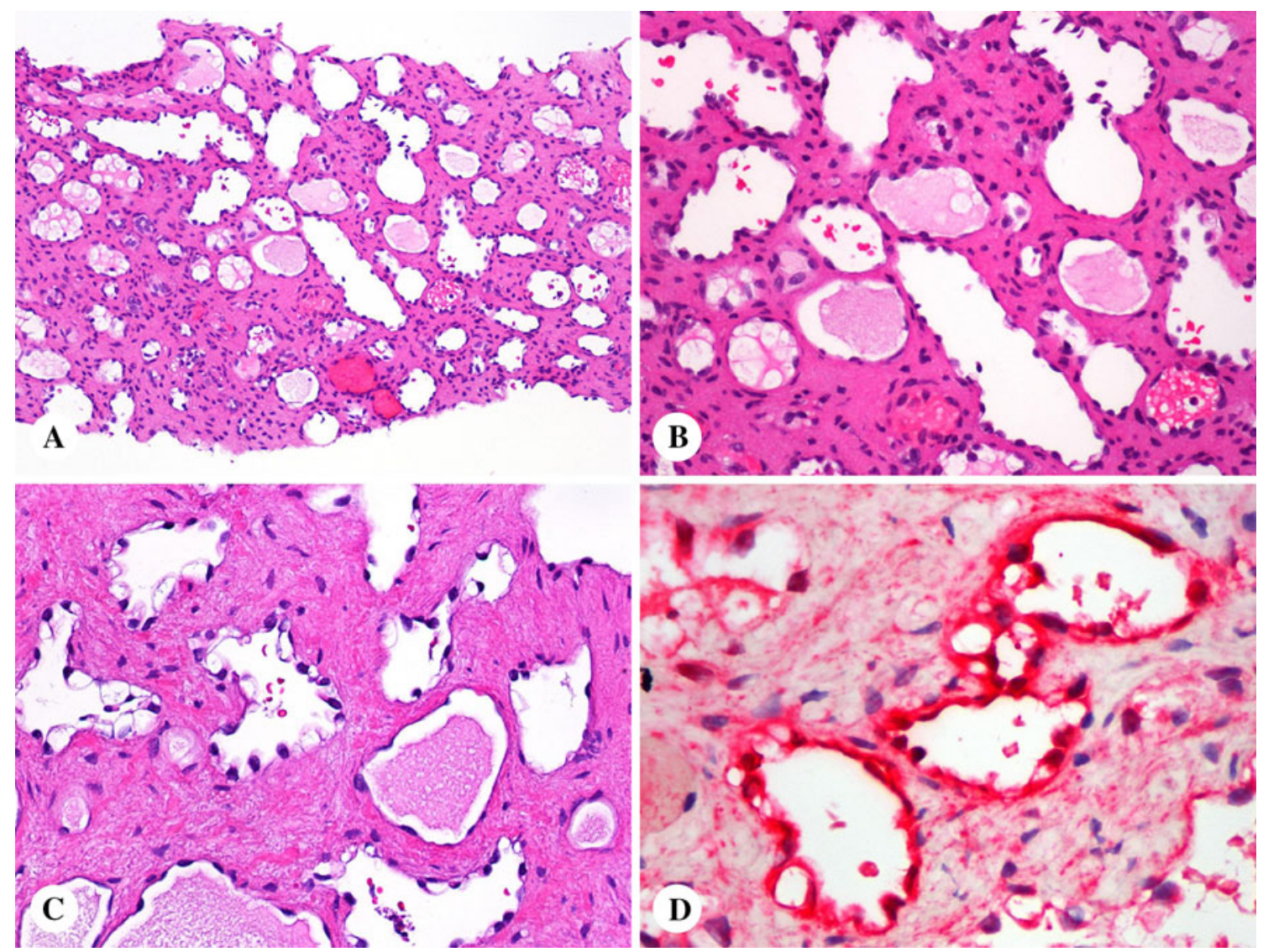

Fig. 3 Lymphangiomatosis involving spleen and liver. a, b Lymphangiomatosis involving the spleen shows parenchymal replacement by vascular proliferation. The lumina are lined by attenuated endothelial cells and contain variably proteinaceous material.

c Lymphangiomatosis involving the liver contains vascular profiles in fibrous stroma. Some profiles have vacuolated inward protuberant ("hobnail") endothelia. d The endothelial cells are immunohistochemically positive for podoplanin (red chromogen)

demonstrated stabilization after administration of pazopanib, known to have antiangiogenic effects, in keeping with the above-mentioned case reports showing indications of clinical benefit with VEGF inhibitors in patients with symptomatic lymphangiomatosis.

Certainly, no general conclusions regarding treatment can be made from this case presentation, especially given that lymphangiomatosis is a usually benign entity that does not cause systemic issues. It is likely that this entity, like many tumors, demonstrates a spectrum of aggression, with some tumors progressing more rapidly than

others and rarely transforming into a life threatening condition. In patients with lymphangiomatosis that is symptomatic or spreading, careful attention to disease "tempo", or the rate of spread based on imaging and/or clinical decline would play an important role in any decisions to institute systemic treatment. Without additional data, treatment and monitoring decisions for these patients must currently be made on a case by case basis with prudent scientific and clinical consideration. Judicious use of antiangiogenic agents is particularly important since antiangiogenic agents as a class may produce 
side effects such as hypertension, proteinuria, impaired wound healing, and events of life threatening vascular compromise [19]. One possible solution is the modification of existing or future phase I clinical trials of antiangiogenic agents to allow the inclusion of patients afflicted by lymphangiomatosis or giant hemangiomas who are symptomatic and afflicted by progressive disease or disease causing deterioration in the patient's quality of life.

\section{CONCLUSION}

The authors report above a case of an adult with lymphangiomatosis involving multiple organs treated with pazopanib with suggestion of clinical benefit. In the face of this mysterious, usually benign disease process which may rarely exhibit aggressive behavior, the authors encourage reports of additional cases to add to the scant literature on this subject and assist in future diagnostic and therapeutic dilemmas.

\section{ACKNOWLEDGMENTS}

No funding or sponsorship was received for this study or publication of this article. Kristen R. Spencer is the guarantor for this article, and takes responsibility for the integrity of the work as a whole.

Conflict of interest. Kristen R. Spencer, Markku M. Miettinen and Janice M. Mehnert declare that they have no conflict of interest. Robert G. Maki is a consultant for GlaxoSmithKline.

Compliance with ethics guidelines. Informed consent was obtained from the patient for being included in the study.
Open Access. This article is distributed under the terms of the Creative Commons Attribution Noncommercial License which permits any noncommercial use, distribution, and reproduction in any medium, provided the original author(s) and the source are credited.

\section{REFERENCES}

1. Aman J, Thunnissen E, Paul MA, van Nieuw Amerongen GP, Vonk-Noordegraaf A. Successful treatment of diffuse pulmonary lymphangiomatosis with bevacizumab. Ann Intern Med. 2012;156: 839-40.

2. Datz C, Graziadei IW, Dietze O, et al. Massive progression of diffuse hepatic lymphangiomatosis after liver resection and rapid deterioration after liver transplantation. Am J Gastroenterol. 2001;96: 1278-81.

3. Huaringa A, Chittari L, Herzog C, Fanning C, Haro M, Eftekhari F. Pleuro-pulmonary lymphangiomatosis: malignant behavior of a benign disease. Internet J Pulm Med. 2005;5.

4. Qutub W, Lewis K, Gonzalez R, Quaife R, Russ P, McCarter M. Lymphangiomatosis masquerading as metastatic melanoma. Am Surg. 2006;72(4):367-70.

5. Reinglas J, Ramphal R, Bromwich M. The successful management of diffuse lymphangiomatosis using sirolimus: a case report. Laryngoscope. 2011;121(9): 1851-4.

6. Chen YL, Lee CC, Yeh ML, Lee JS, Sung TC. Generalized lymphangiomatosis presenting as cardiomegaly. J Formos Med Assoc. 2007;106(3): S10-4.

7. Karmody CS, Fortson JK, Calcaterra VE. Lymphangiomas of the head and neck in adults. Otolaryngol Head Neck Surg. 1982;90(3 Pt 1): 283-8.

8. Reinhardt MA, Nelson SC, Sencer SF, Bostrom BC, Kurachek SC, Nesbit ME. Treatment of childhood lymphangiomas with interferon-alpha. J Pediatr Hematol Oncol. 1997;19(3):232-6.

9. Turner C, Gross S. Treatment of recurrent suprahyoid cervicofacial lymphangioma with intravenous cyclophosphamide. Am J Pediatr Hematol Oncol. 1994;16(4):325-8. 
10. Hellman JR, Myer CM, Prenger EC. Therapeutic alternatives in the treatment of life-threatening vasoformative tumors. Am J Otolaryngol. 1992;13(1):48-53.

11. Tilg H. New insights into the mechanisms of interferon alfa: an immuno-regulatory and anti-inflammatory cytokine. Gastroenterology. 1997;112:1017-21.

12. Tazelaar HD, Kerr D, Yousem SA, Saldana MJ, Langston C, Colby TV. Diffuse pulmonary lymphangiomatosis. Hum Pathol. 1993;24(12): 1313-22.

13. Margraf LR. Thoracic lymphangiomatosis. Pediatr Pathol Lab Med. 1996;16(1):155-60.

14. Laverdiere C, David M, Dubois J, Russo P, Hershon L, Lapierre JG. Improvement of disseminated lymphangiomatosis with recombinant interferon therapy. Pediatr Pulmonol. 2000;29(4):321-4.
15. Vial T, Descotes J. Clinical toxicity of the interferons. Drug Saf. 1994;10(2):115-50.

16. Kasper B, Hohenberger P. Pazopanib: a promising new agent in the treatment of soft tissue sarcomas. Future Oncol. 2011;7(12):1373-83.

17. Fernandez S, Cook GW, Arber DA. Metastasizing splenic littoral cell hemangioendothelioma. Am J Surg Pathol. 2006;30(8):1036-40.

18. Mills SE. The spleen. In: Mills SE, et al., editors. Sternberg's diagnostic surgical pathology. 5th ed. Philadelphia: Lippincott, Williams and Wilkins; 2010. p. $745-71$.

19. Chen HX, Cleck JN. Adverse effects of anticancer agents that target the VEGF pathway. Nat Rev Clin Oncol. 2009;6(8):465-77. 